\title{
Response of HCV Yemeni Patients to Sofosbuvir/Ledipasvir in Combination with Ribavirin
}

\author{
Abdulgafoor Kassim¹, Ramea Alathwary ${ }^{2 *}$, Talal Al-hogami ${ }^{3}$ \\ ${ }^{1}$ Gastroenterology and Hepatology, Internal Medicine, Faculty of Medicine, Taiz University, Taiz, Yemen \\ ${ }^{2}$ Internal Medicine, Internal Medicine Department, Faculty of Medicine, Taiz University, Taiz, Yemen \\ ${ }^{3}$ Alfa Medical Lab, Ibb, Yemen \\ Email: a.algafoor@gmail.com, *alathwaryramea@gmail.com
}

How to cite this paper: Kassim, A., Alathwary, R. and Al-hogami, T. (2019) Response of HCV Yemeni Patients to Sofosbuvir/Ledipasvir in Combination with Ribavirin. Open Access Library Journal, 6: e5507.

https://doi.org/10.4236/oalib.1105507

Received: May 27, 2019

Accepted: June 25, 2019

Published: June 28, 2019

Copyright $\odot 2019$ by author(s) and Open Access Library Inc.

This work is licensed under the Creative Commons Attribution International License (CC BY 4.0).

http://creativecommons.org/licenses/by/4.0/

\section{(c) (i) Open Access}

\begin{abstract}
Objectives: To study the effectiveness and safety of ledipasvir-sofosbuvir plus ribavirin treatment in Yemeni patient with HCV infection. Design: Prospective study of sixty-five Yemeni patients confirmed with HCV infection during the period from January 2017 to April 2018. Setting: Gastrointestinal and liver diseases specialized center in Ibb city, Yemen. Subjects: Patients with proved HCV infection by PCR. Results: We collected 65 cases with HCV infection. They were $19(29.2 \%)$ males and 46 (70.8) females with age ranged between 18 years and 70 years and the mean age was $47.98 \pm 11.90$ years. sixteen $(24.6 \%)$ of them had compensated liver cirrhosis and the remainder were non-cirrhotic healthy individual. The early virological response reported in $100 \%$ of cases while the sustained virological response (SVR) reported in $90.8 \%$. The reported side effects of our patients were fatigue in (24.6\%), abdominal pain in $(13.8 \%)$, diarrhea in $(10.8 \%)$, insomnia in $(9.2 \%)$, headache in $(7.7 \%)$, and nausea/vomiting in (7.7\%). Conclusion: The Sofosbuvir-ledipasvir plus Ribavirin treatment was highly effective and safe for the treatment of hepatitis $\mathrm{C}$ patients in Yemen.
\end{abstract}

\section{Subject Areas}

Gastroenterology \& Hepatology

Keywords

HCV, Early Virological Response (EVR), MPIVIROPACT Plus, Yemen

\section{Introduction}

Hepatitis $\mathrm{C}$ virus (HCV) is one of the major globally causes of morbidity and 
mortality [1]. It is the $7^{\text {th }}$ leading cause of death worldwide (World Health Organization, 2017) [2]. Chronic HCV infection is often associated with the development of liver cirrhosis, hepatocellular cancer, and liver failure [1] [3]. A recent estimate showed an increase in its seroprevalence over the last decade to $2.8 \%$, corresponding to more than 185 million infections worldwide [4]. In all the cases, studies focuse only on the presence of HCV antibodies which generally overestimate the disease burden because they include also patients healed spontaneously or by treatments [5]. The geographical distribution of this infection appears to vary from one region to another [6]. The Middle East and North Africa (MENA) region appears to have the highest HCV prevalence worldwide with Egypt recording the highest national prevalence in the adult population at $14.7 \%$, and a somewhat lower prevalence $(>0.1 \%)$ is reported in Western Europe, including the United Kingdom, Finland, and Germany [1] [6] [7] [8]. In our country the prevalence ranges between $0.6 \%-5.1 \%$ in different cities of the country [9]. Up to now, HCV has seven major genotypes and 86 confirmed subtypes [1] [8]. In addition to heterogeneity in the prevalence of infection there is also heterogeneity in the distribution of viral genotypes. In most developed countries, genotypes 1 and 3 dominate whereas genotypes 4, 5, and 6 are more common in countries with limited healthcare resources including Middle East [10]. Genotype 4 is the most common genotype in Middle East countries including Yemen in both males and females accounting for $63.7 \%$ of all HCV infection [11] [12]. Up to the end of 2013, Interferon given by subcutaneous injection once weekly, and oral Ribavirin twice daily for 24 - 48 weeks were the recommended treatment option for Hepatitis $\mathrm{C}$ patients and achieved a sustained virologic response (SVR) rate in $48 \%-55 \%$ and relapse rate in $24 \%$ [1] [8] [13]. In the past few years, new treatment regimens have been developed including directly acting antiviral (DAA) [1] [8]. The first HCV nucleotide polymerase inhibitor (PI), Sofosbuvir (SOF), was approved for use for genotypes 1, 2, 3, and 4 [14]. In 2014, the single-tablet regimen of SOF combined with the NS5A inhibitor ledipasvir (LDV) was approved for patients with HCV [15]. HCV treatment using combinations of directly acting antiviral agents has led to a great increase in the number of patients achieving sustained virological response (SVR) rates, reaching up to $90 \%$ - 95\% and even higher [8] [16]. These regimens markedly improved efficacy and tolerability and the most common adverse effects reported with SOF/LDV combination were headache and fatigue [1] [8] [17]. Therefore, these newly approved agents are recommended as first-line therapy for all indications throughout the guidelines [1] [8]. With the new Interferon free DAAs regimens for HCV treatment, still Ribavirin is needed in many schedules specially with PI and NS5A Inhibitors [18]. Although the mechanism of its action is not well understood, it is needed to enhance the response rate and decrease the relapse rate in addition to shorting the treatment duration as in patients with cirrhosis [18]. The sofosbuvir/ledipasvir (SOF/LDV) combination is available in a two-drug fixed-dose tablet containing sofosbuvir $400 \mathrm{mg}$ and ledipasvir $90 \mathrm{mg}$. It has been recently approved for the treatment of genotypes 1, 4, 
5 , and 6 as a once-daily 12 -week regimen for treatment of non-cirrhotic patients or patients with compensated cirrhosis (Child-Pugh A) [19]. The response rate of the therapy was dependent upon a number of host and viral factors including patient age, liver condition, viral load and HCV genotype etc. [1] [8].

This is the first study in our country which evaluate the efficacy, tolerability, and safety of ledipasvir and sofosbuvir plus fixed-dose ribavirin for treatment of patients infected with HCV, with or without cirrhosis. We also analyzed different host and viral parameters which may affect the treatment response.

\section{Patients and Methods}

This study was conducted in a center of gastrointestinal and liver diseases in Ibb city in the period between January 2017 to April 2018. For every patient with positive HCV Ab by ELIZA (using Cobas e 411-Roche), a comprehensive history, physical examination, and standard lab tests including; complete blood picture, liver function tests, renal function tests, blood sugar, PT, INR, serum albumin, HIV and HBs Ag were done. Abdominal ultrasound was carried out by an expert sonographer to assess the liver status including size, architecture, surface and presence of focal lesions. Spleen size, portal vein diameter and presence of ascites were also evaluated. An upper GI endoscopy (using PENTAX-EPK-5000 Unit) was performed to all patients to assess presence or absence of esophageal varices, grading of esophageal varices if present, and presence or absence of gastropathy. HCV infection was confirmed by PCR (using Cobas Taqman 48 -Roche) with the limit of detection $<18$ copies $/ \mathrm{ml}$.

The inclusion criteria in our study were patients with PCR confirmed HCV infection; with either normal liver or with compensated CLD (Child Pugh score A) who accepted to be enrolled in the study by giving a written or verbal consent. The exclusion criteria were; patients less than 18 years old, decompensated liver disease (CTP score B and C), presence of hepatic focal lesion/s (HCC), concomitant HBV infection, and severe comorbidities; renal failure, cardiac failure, and advanced malignancy. Those who were previously treated by the recent antiviral treatment and failed to respond or relapsed were also excluded. The enrolled patients were divided into two groups; those with no clinical or para-clinical manifestations of CLD as non-cirrhotic group and those with clini$\mathrm{cal}$ and para-clinical findings that are consistent with chronic liver disease as cirrhotic group. A combination of a fixed-dose tablet of ledipasvir and sofosbuvir $(90 \mathrm{mg} / 400 \mathrm{mg}$ ) (MPIVIROPCK PLUS, Marcyl pharmaceutical industries, Egypt) once daily plus fixed dose ribavirin $800 \mathrm{mg}$ (Ribazole-GetZ pharma (pvt.) Limited, Pakistan) in a divided dose twice daily for 12 weeks were given. Clinical and para-clinical assessment was carried out monthly and enquiring for any side effects of the drugs was done. HCV RNA by PCR was requested after completion the treatment and 3 months later. Negative result after completion of the treatment is defined as early virological response (EVR) and negative result after 3 months is considered a sustained viral response (SVR). 
Unfortunately, genotyping was not done because of unavailability of this test in our country. Moreover; sending of blood sample for genotyping outside the country was not possible due to difficulty in transportation caused by war and siege in the area.

SPSS version 21 (SPSS, Inc., Chicago, IL, USA) was used to analyze the data. The mean, standard deviation and ranges were used for quantitative variables and percentages were used for qualitative variables. The $\mathrm{p}$-value was calculated by Chi-square test and considered significant if it is $<0.05$.

\section{Results}

We studied sixty-five cases of confirmed HCV infection, 19(29.2\%) were males and $46(70.8 \%)$ were females with age ranged between 18 years and 70 years and the mean age was $47.98 \pm 11.90$ years. The mean age for males was of $46.89 \pm$ 15.08 and was of $48.43 \pm 10.48$ for females. Male to female ratio was 1:2.4 (Table 1). Sixteen (24.6\%) of our patients had compensated liver cirrhosis and the remainder were non-cirrhotic healthy individuals.

Clinical and biochemical characteristics are shown in (Table 2).

Response to the treatment and relapse rate in both cirrhotic and non-cirrhotic groups are studied in (Table 3).

The most frequent side effects and laboratory abnormalities of the treatment are reported in (Table 4 and Table 5).

Finally, we studied some host and viral parameters that may affect the treatment response in our patients and the only significant differences seen in correlation with liver condition as $\mathrm{P}$ value $<0.05$ (Table 6).

Table 1. Demographic characteristics of our studied cases $($ no $=65)$.

\begin{tabular}{rlcc}
\hline Characteristics & No. & $(\%)$ \\
\hline Age & & & \\
& $-18-39$ & 17 & 22 \\
& $-40-60$ & 36 & 55.4 \\
& $->60$ & 12 & 18.5 \\
Sex & & & \\
& - Male & 19 & 29.2 \\
& - Female & 46 & 70.8 \\
\hline
\end{tabular}

Table 2. Clinical and biochemical characteristics of our studied cases (no $=65)$.

\begin{tabular}{cc}
\hline Characteristics & No. $(\%)$ \\
\hline Liver condition & \\
- Cirrhotic & $16(24.6)$ \\
- Non-cirrhotic & $49(75.4)$ \\
Mean HCV RNA, log10 IU/mL & $($ Mean \pm SD) \\
- In cirrhotic patient & $7,160,929 \pm 22,023,603$ \\
- In non-cirrhotic cases & $3,252,136 \pm 5,632,551$ \\
Biochemical profile & $($ Mean $\pm \mathrm{SD})$ \\
\hline
\end{tabular}




\section{Continued}

\begin{tabular}{cc}
\hline Hb & $13.26 \pm 1.7$ \\
WBC & $5892 \pm 1481$ \\
Platelets & $209,885.92 \pm 115,162.35$ \\
Total bilirubin & $1.42 \pm 1.35$ \\
ALT & $61.13 \pm 66.32$ \\
Albumin & $3.65 \pm 0.48$ \\
\hline
\end{tabular}

$\mathrm{Hb}=$ Hemoglobin, $\mathrm{WBC}=$ White Blood Cell, $\mathrm{ALT}=$ alanine aminotransferase, $\mathrm{PT}=$ Prothrombin Time.

Table 3. Treatment response in cirrhotic and non-cirrhotic patients $($ no $=65)$.

\begin{tabular}{ccccccc}
\hline Treatment response & \multicolumn{2}{c}{ Cirrhotic $($ no $=16)$} \\
& No. & $(\%)$ & $\begin{array}{r}\text { Non-cirrhotic }(\text { no }=49) \\
(\%)\end{array}$ & \multicolumn{2}{c}{ Total $($ no $=65)$} \\
EVR at 12 weeks & 16 & 100 & 49 & 100 & 65 & 100 \\
SVR at 24 weeks & 13 & $\mathbf{8 1 . 3}$ & 46 & 93.9 & 59 & 90.8 \\
Relapse & 3 & 18.7 & 3 & 6.1 & 6 & 9.2 \\
\hline
\end{tabular}

$\mathrm{EVR}=$ Early virological response, $\mathrm{SVR}=$ Sustained virological response.

Table 4. Adverse effects and laboratory abnormalities of the treatment in our cases (no = 65).

\begin{tabular}{ccc}
\hline Side effects & No & $(\%)$ \\
\hline Fatigue & 16 & 24.6 \\
Abdominal pain & 9 & 13.8 \\
Diarrhea & 7 & 10.8 \\
Insomnia & 6 & 9.2 \\
Nausea & 5 & 7.7 \\
Headache & 5 & 7.7 \\
\hline
\end{tabular}

Table 5. Abnormality in the biochemical profile before and after the treatment (no $=65)$.

\begin{tabular}{cccccccc}
\hline \multirow{2}{*}{ Biochemical test } & \multicolumn{5}{c}{ Before } & \multicolumn{4}{c}{ After } & P value \\
\cline { 2 - 7 } & Non-cirrhotic & Cirrhotic & Total & Non-cirrhotic & Cirrhotic & Total & \\
\hline Elevated ALT & $20(40.8)$ & $12(75.0)$ & $32(49.2)$ & $18(36.7)$ & $12(75.0)$ & $30(46.2)$ & 0.83 \\
Hb $<12$ gm\% & $7(14.3)$ & $5(31.2)$ & $12(18.5)$ & $10(20.4)$ & $6(37.5)$ & $16(24.8)$ & 0.16 \\
Leukopenia & $3(6.1)$ & $1(6.3)$ & $4(6.2)$ & $1(2)$ & $6(37.5)$ & $7(10.8)$ & 0.90 \\
Thrombocytopenia & $5(10.2)$ & $11(68.8)$ & $16(24.6)$ & $5(10.2)$ & $13(81.3)$ & $18(27.7)$ & 0.32 \\
$\begin{array}{c}\text { Elevated total } \\
\text { bilirubin }\end{array}$ & $15(30.6)$ & $8(50)$ & $23(35.4)$ & $10(20.8)$ & $12(75)$ & $22(34.4)$ & 0.15
\end{tabular}

Table 6. Association of different variables in achieving sustained viral response in HCV patients.

\begin{tabular}{ccccccc}
\hline \multirow{2}{*}{ Variables } & \multicolumn{2}{c}{ Viral load } & \multicolumn{2}{c}{ Age } & \multicolumn{2}{c}{ Liver condition } \\
\cline { 2 - 7 } Treatment response & $<8 \times 10^{5}$ & $>8 \times 10^{5}$ & $<40$ & $>40$ & Non-cirrhotic & Cirrhotic \\
\hline SVR at 24 weeks & 94.7 & 85.2 & 100 & 87.5 & 93.9 & 81.3 \\
Relapse & 5.3 & 14.8 & 0 & 12.5 & 6.1 & 18.7 \\
P value & \multicolumn{2}{c}{0.19} & & 0.12 & & 0.04 \\
\hline
\end{tabular}




\section{Discussion}

Hepatitis $\mathrm{C}$ virus (HCV) infection is a global public health problem. Approximately 130 to 170 million people experience chronic HCV infection, which has a global prevalence of $2 \%-3 \%$ [20]. Treatment of hepatitis $\mathrm{C}$ has improved considerably over the last 10 years since the introduction of Sofosbuvir, as NS5B polymerase inhibitor with potent activity against all $6 \mathrm{HCV}$ genotypes (pangenotypic activity) [21]. AASLD in 2016 and EASL 2015 issued many guidelines for HCV treatment including the combination of Sofosbuvir and ledipasvir with or without Ribavirin for 12 weeks [22] [23]. Nowadays, despite remarkable progress in the treatment of chronic HCV infection, treatment in our country remains a clinical challenge due to poverty, unavailability of most recent and newly studied drugs. Moreover, the recent drugs are highly expensive and out of reach for most of our patients. This study evaluated for the first time in our country the efficacy and safety of ledipasvir-sofosbuvir plus Ribavirin for 12 weeks in patients with HCV infection as these drugs are the most available and cheaper one.

We studied sixty five cases of confirmed HCV infection, 19 (29.2\%) were males and 46 (70.8\%) were females. Male to female ratio was 1:2.4, and this is in concordance with some studies in our country and some neighboring countries were the prevalence of HCV infection was seen more in females than males [24] [25] [26] [27]. Meanwhile in many studies from different parts of the world the infection is more common in males [28] [29]. This difference of HCV prevalence in both gender could be explained by different ways of HCV transmission. One of the explanation of the increase HCV infection among females in our community may be understood in context of high exposure of females owing to high productivity; the infertility rate in Yemen is 3.6 [30].

The mean age was of $47.98 \pm 11.90$ years with a range between $18-70$ years. The non-cirrhotic group represents 49 (75.4\%) and the cirrhotic one represents $16(24.6 \%)$ and this is in concordance with most of the studies [28] [30] [31]. According to WHO and few studies in our country the most common HCV genotype is type 4 followed by type 1 [5] [24]. Genotyping was not done in our study due to unavailability of the test in our country and difficulty of sending blood sample outside the country due to war. The 12-week regimen of ledipasvir-sofosbuvir with ribavirin was highly effective in treatment of our patients, with an EVR of $100 \%$, overall SVR rate of $90.8 \%$ and relapse rate of $9.2 \%$. The effectiveness of these combinations among non-cirrhotic patients was of 93.9\%; Meanwhile, in cirrhotic patients it represented only $81.3 \%$ ( $\mathrm{P}$ value $=$ 0.049). These results are comparable to those reported by many studies from neighboring countries with similar HCV genotype distributions [32] [33]. A study done by Feld et al., showed similar result despite using of this regimen for different HCV genotypes (genotype 3) this could be explained by the potency of ledipasvir in combination with other DAA against all HCV genotypes [29].

According to the global literatures and many studies, the response rate of the 
therapy is dependent upon a number of host and viral factors including patient age, liver condition, viral load and HCV genotype etc [1] [8] [34]. We studied the impact of some of these factors on treatment outcome of our patients (Table 6). The SVR in patients aged $<40$ years was of $100 \%$ and in age $>40$ years was of $87.8 \%$. The response in patients with viral load below $8 \times 10^{5}$ was of $94.7 \%$ and in those with viral load above $8 \times 10^{5}$ was of $85.2 \%$. Moreover, the SVR among non-cirrhotic and cirrhotic patients was of $93.9 \%$ and $81.3 \%$ respectively. The only statistically significant differences were found in correlation with liver condition $(P$ value $=0.04)($ Table 6$)$. Absence of statistically significant differences for the other factors in our study could be due to small sample size. Concerning safety and tolerability of this combination, no significant clinical or biochemical adverse effects were reported, and the most common side effects were fatigue, nausea and headache in (15.4\%), (6.4\%) and (8\%) respectively. No statistically significant biochemical differences before and after taking the drugs were reported (Table 5). These results are similar to the reported data in the global literatures and most of the recent studies [1] [8] [34].

The limitations of our study include the relatively small number of some subgroup of patients such as patients with cirrhosis. Another limitation was absence of genotyping in our study due to inability to perform the test in our country at the present time.

\section{Conclusion}

The combination of ledipasvir-sofosbuvir plus fixed-dose ribavirin was effective and safe in treatment of HCV infection among Yemeni patients with overall SVR12 of $90.7 \%$ and no clinically significant side effects.

\section{Conflicts of Interest}

The authors declare no conflicts of interest regarding the publication of this paper.

\section{References}

[1] James, S.D., Anna, S.F.L., Guadalupe, G.T. and Massimo, P. (2018) Sherlock's Diseases of the Liver and Biliary System. 13th Edition, Vol. 1, John Wiley \& Sons Ltd., Oxford, 436-459.

[2] World Health Organization (2017) Global Hepatitis Report 2017. http://apps.who.int/iris/bitstream/handle/10665/255016/9789241565455-eng.pdf;jse ssionid=E2D0796013167FC7FFA0295C58EF490D? sequence $=1$

[3] The Global Burden of Hepatitis C Working Group (2004) Global Burden of Disease (GBD) for Hepatitis C. The Journal of Clinical Pharmacology, 44, 20-29. https://doi.org/10.1177/0091270003258669

[4] Mohd Hanafiah, K., Groeger, J., Flaxman, A.D. and Wiersma, S.T. (2013) Global Epidemiology of Hepatitis C Virus Infection: New Estimates of Age-Specific Antibody to HCV Seroprevalence. Hepatology, 57, 1333-1342. https://doi.org/10.1002/hep.26141

[5] Petruzziello, A., Marigliano, S., Loquercio, G., Cozzolino, A. and Cacciapuoti, C. 
(2016) Global Epidemiology of Hepatitis C Virus Infection: An Up-Date of the Distribution and Circulation of Hepatitis C Virus Genotypes. World Journal of Gastroenterology, 22, 7824-7840. https://doi.org/10.3748/wjg.v22.i34.7824

[6] Lavanchy, D. (2011) Evolving Epidemiology of Hepatitis C Virus. Clinical Microbiology and Infection: The Official Publication of the European Society of Clinical Microbiology and Infectious Diseases, 17, 107-115. https://doi.org/10.1111/j.1469-0691.2010.03432.x

[7] Desenclos, J.C. (2003) The Challenge of Hepatitis C Surveillance in Europe. Eurosurveillance, 8, 99-100. https://doi.org/10.2807/esm.08.05.00409-en

[8] Dennis, L.K., Stephen, L.H., Larry, J., Anthony, S.F., Dan, L.L. and Joseph, L. (2015) Harrison's Principle of Internal Medicine. 19th Edition, Vol. 2, McGraw-Hill Education, New York, 2041-2052.

[9] Rajesh, G. and Sadiq, A. (2012) Epidemiology of Viral Hepatitis B and C Infections in Ibb City, Yemen. Hepatitis Monthly, 12, 460-462.

[10] Thursz, M. and Fontanet, A. (2014) HCV Transmission in Industrialized Countries and Resource-Constrained Areas. Nature Reviews Gastroenterology \& Hepatology, 11, 28-35. https://doi.org/10.1038/nrgastro.2013.179

[11] Blach, S., Zeuzem, S., Manns, M., Altraif, I., Duberg, A.S., Muljono, D.H., et al. (2017) Global Prevalence and Genotype Distribution of Hepatitis C Virus Infection in 2015: A Modeling Study. The Lancet Gastroenterology \& Hepatology, 2, 161-176. https://doi.org/10.1016/S2468-1253(16)30181-9

[12] Al-Shamahy, H.A. and Abdu, S.S.A. (2013) Genotyping of Hepatitis C Virus (HCV) in Infected Patients from Yemen. European Journal of Basic Medical Science, 3, 78-82.

[13] Zoulim, F., Liang, T.J., Gerbes, A.L., et al. (2015) Hepatitis C Virus Treatment in the Real World: Optimizing Treatment and Access to Therapies. Gut, 64, 1824-1833. https://doi.org/10.1136/gutjnl-2015-310421

[14] Cheng, G., Tian, Y., Doehle, B., et al. (2016) In Vitro Antiviral Activity and Resistance Profile Characterization of the Hepatitis C Virus NS5A Inhibitor Ledipasvir. Antimicrobial Agents and Chemotherapy, 60, 1847-1853.

https://doi.org/10.1128/AAC.02524-15

[15] Gower, E., Estes, C., Blach, S., Razavi-Shearer, K. and Razavi, H. (2014) Global Epidemiology and Genotype Distribution of the Hepatitis C Virus Infection. Journal of Hepatology, 61, S45-S57. https://doi.org/10.1016/j.jhep.2014.07.027

[16] Lam, A.M., Espiritu, C., Bansal, S., Micolochick Steuer, H.M., Niu, C., Zennou, V., Keilman, M., Zhu, Y., Lan, S., Otto, M.J. and Furman, P.A. (2012) Genotype and Subtype Profiling of PSI-7977 as a Nucleotide Inhibitor of Hepatitis C Virus. Antimicrobial Agents and Chemotherapy, 56, 3359-3368. https://doi.org/10.1128/AAC.00054-12

[17] Nelson, D.R., Cooper, J.N., Lalezari, J.P., Lawitz, E., Pockros, P.J., et al. (2015) All-Oral 12-Week Treatment with Daclatasvir plus Sofosbuvir in Patients with Hepatitis C Virus Genotype 3 Infection: ALLY-3 Phase III Study. Hepatology, 61, 1127-1135. https://doi.org/10.1002/hep.27726

[18] Hosny, S., Abdel, R.Z., Eman, M., Zienab, Z., Hend, S., Sherin, A.A. and Mervat, A.A. (2016) Sofosbuvir plus Daclatasvir with Fixed versus Weight Adjusted Dose of Ribavirin for Treatment of HCV, Genotype 4 among Egyptian Patients. EC Gastroenterology and Digestive System, 1, 143-153.

[19] European Association for the Study of the Liver (2018) EASL Recommendations on 
Treatment of Hepatitis C 2018. Journal of Hepatology, 69, 461-511. https://doi.org/10.1016/j.jhep.2018.03.026

[20] Lavanchy, D. (2009) The Global Burden of Hepatitis C. Liver International, 29, 74-81. https://doi.org/10.1111/j.1478-3231.2008.01934.x

[21] Koff, R.S. (2014) Review Article: The Efficacy and Safety of Sofosbuvir, a Novel, Oral Nucleotide NS5B Polymerase Inhibitor, in the Treatment of Chronic Hepatitis C Virus infection. Alimentary Pharmacology \& Therapeutics, 39, 478-487. https://doi.org/10.1016/j.jhep.2016.09.001

[22] European Association for the Study of the Liver (2017) EASL Recommendations on Treatment of Hepatitis C 2016. Journal of Hepatology, 66, 153-194.

[23] Basu, P., et al. (2015) Sofosbuvir and Ledipasvir versus Sofosbuvir and Simeprevir Combination Therapy in the Management of Acute Hepatitis C: A Randomized Open Label Prospective Clinical Pilot Study. SLAM C Study. AASLD 2015, San Francisco, 13-17 November 2015, 1-20.

[24] Rajesh, N.G. and Sadiq, K.A. (2012) Seroprevalence and Risk Factors for Hepatitis C Virus Infection among General Population in Central Region of Yemen. Hepatitis Research and Treatment, 2012, Article ID: 689726. https://doi.org/10.1155/2012/689726

[25] Ali, A., Ahmad, H., Ali, I., Khan, S., Zaidi, G. and Idrees, M. (2010) Prevalence of Active Hepatitis C Virus Infection in District Mansehra Pakistan. Virology Journal, 7, 334. https://doi.org/10.1186/1743-422X-7-334

[26] Chen, S.L. and Morgan, T.R. (2006) The Natural History of Hepatitis C Virus, (HCV) Infection. International Journal Medical Science, 3, 47-52. https://doi.org/10.7150/ijms.3.47

[27] Erensoy, S. (2010) Burden and Prevention of Viral Hepatitis in Turkey, Istanbul, Turkey, November 12-13, 2009. Meeting News, 18, 2.

[28] Kamal, S.M. (2008) Acute Hepatitis C: A Systematic Review. American Journal of Gastroenterology, 103, 1283-1297. https://doi.org/10.1111/j.1572-0241.2008.01825.x

[29] Feld, J.J., Alnoor, R., Stephen, D.S., et al. (2017) Ledipasvir-Sofosbuvir plus Ribavirin in Treatment-Naive Patients with Hepatitis C Virus Genotype 3 Infection: An Open-Label Study. Clinical Infectious Diseases, 65, 13-19. https://doi.org/10.1093/cid/cix289

[30] https://www.indexmundi.com/yemen/total fertility rate.html

[31] Zubia, J., Yasir, W., Maryam, M. and Asghar, A.D. (2018) Effect of Sofosbuvir plus Ribavirin Therapy on Hepatitis C Patients in Pakistan: A Retrospective Study. Peer $J, 6, \mathrm{e} 4853$.

[32] Hend, I.S., Karim, A., Sherif, R., Eman, M. and Gamal, E. (2018) Generic Sofosbuvir/Ledipasvir for Treatment of Naïve, Non-Cirrhotic, Easy to Treat Patients with Chronic Hepatitis C Genotype 4: 8 vs. 12 Weeks of Treatment. Hepatitis Monthly, 18, e78777. https://doi.org/10.5812/hepatmon.78777

[33] Shiha, G., Esmat, G., Hassany, M., Soliman, R., et al. (2019) Ledipasvir/Sofosbuvir with or without Ribavirin for 8 or 12 Weeks for the Treatment of HCV Genotype 4 Infection: Results from a Randomised Phase III Study in Egypt. Gut, 68, 721-728. https://doi.org/10.1136/gutjnl-2017-315906

[34] Waheed, Y. (2015) Effect of Interferon plus Ribavirin Therapy on Hepatitis C Virus Genotype 3 Patients from Pakistan: Treatment Response, Side Effects and Future Prospective. Asian Pacific Journal of Tropical Medicine, 8, 85-89. https://doi.org/10.1016/S1995-7645(14)60193-0 